\title{
When matter matters
}

\author{
Damien A. Easson, ${ }^{a}$ Ignacy Sawicki ${ }^{b}$ and Alexander Vikman ${ }^{c, d}$ \\ ${ }^{a}$ Department of Physics \& School of Earth and Space Exploration \& Beyond Center, \\ Arizona State University, Tempe, AZ, 85287-1504, USA \\ ${ }^{b}$ Institut für Theoretische Physik, Ruprecht-Karls-Universität Heidelberg \\ Philosophenweg 16, 69120 Heidelberg, Germany \\ ${ }^{c}$ CERN, Theory Division, CH-1211 Genève 23, Switzerland \\ ${ }^{d}$ Stanford University, Department of Physics, Stanford, CA 94305, USA \\ E-mail: easson@asu.edu, ignacy.sawicki@uni-heidelberg.de, alexander.vikman@cern.ch
}

\begin{abstract}
We study a recently proposed scenario for the early universe: Subluminal Galilean Genesis. We prove that without any other matter present in the spatially flat Friedmann universe, the perturbations of the Galileon scalar field propagate with a speed at most equal to the speed of light. This proof applies to all cosmological solutions - to the whole phase space. However, in a more realistic situation, when one includes any matter which is not directly coupled to the Galileon, there always exists a region of phase space where these perturbations propagate superluminally, indeed with arbitrarily high speed. We illustrate our analytic proof with numerical computations. We discuss the implications of this result for the possible UV completion of the model.
\end{abstract}




\section{Contents}

2 Model and Main Equations

2.1 Some properties of models with Kinetic Gravity Braiding

3 Robust subluminality without external matter $\quad 5$

4 Superluminality with external matter $\quad 8$

$4.1(m, h, \rho)$ coordinates and a proof of general superluminality $\quad 8$

$4.2(\phi, m, \rho)$ coordinates and induced superluminality of original configurations $\quad 9$

5 Results Summary and Discussion

\section{Introduction}

The theory of generation of quantum cosmological perturbations ${ }^{1}$ during early-universe inflation ${ }^{2}$ received additional strong support from the recent results of the Planck collaboration [20]. Inflationary spacetimes usually feature a singularity or in other words strong quantum gravity stage far enough back in their past [21]. For a universe with no spatial curvature, the only known method of avoiding a strong quantum gravity stage is to posit a period in the evolution of the universe where the Null Energy Condition $(\mathrm{NEC})^{3}$ is violated. However, until recently, such classical and local constructions in the context of standard general relativity were always plagued by some sort of pathological instabilitieseither ghosts or gradient instabilities or both, see e.g. [22]. ${ }^{4}$ The situation has changed with the rediscovery [27-35] of more general single scalar-field theories [36], which contain higher derivatives of the scalar field in the action but still have only one scalar degree of freedom. These theories were explicitly demonstrated to be able to violate the NEC with neither ghost nor gradient instabilities in at least a part of their phase space, ${ }^{5}$ see e.g. [33, 38-40]. Moreover, it was shown in [41] that these theories can realise a bounce and avoid a strong quantum-gravity regime but only at the price of a strongly coupled regime of these non-renormalizable theories in the initial, now collapsing, universe.

An interesting and unusual scenario for the initial stages of the universe was proposed in [39]: Galilean Genesis. There, the universe starts from the Minkowski spacetime which corresponds to an unstable and singular configuration of the Galileon scalar field with zero energy density. Small departures from this original state violate the NEC. Thus starting from this configuration the universe can expand by generating the energy density during its evolution. In fact this evolution is just a superinflationary stage with a very small acceleration. ${ }^{6}$ Nearly scale-invariant cosmological perturbations are supposed to be generated by introducing an extra spectator scalar field coupled to the Galileon in such a way that effective metric reproduces quasi de Sitter. Eventually the Galileon should disappear by transmitting its energy into radiation, before the universe runs into a Big Rip singularity [44, 45]. This may happen through a reheating mechanism proposed in [46]. However, it is not clear whether

\footnotetext{
${ }^{1}$ For the scalar (or energy density) perturbations which have been already observed, see [1-10], while for the tensor modes (or gravitational waves), see an earlier work [11].

${ }^{2}$ See $[12-14]$ and $[11,15-19]$ for earlier works on the quasi-de Sitter stage in the early universe. We are thankful to the anonymous referee for drawing our attention to works $[16,19]$.

${ }^{3}$ This condition states that $T^{\mu \nu} n_{\mu} n_{\nu} \geq 0$, where $T^{\mu \nu}$ is an energy-momentum tensor $n^{\mu}$ represents all null vectors.

${ }^{4}$ One may try to avoid these problems by adding higher derivatives directly to the EFT for perturbations see e.g. [23-26]. This approach may be useful for a better systematisation of the perturbative expansion but cannot elucidate the behaviour of the cosmological background.

${ }^{5}$ However, it does not mean that there are no instabilities at all. Indeed, there are good physical arguments [37] that there are more subtle and less dangerous run-away instabilities which are still present in these theories.

${ }^{6}$ This is also the case for other Genesis scenarios $[42,43]$.
} 
one can really get rid of the Galileon, so that it does not spoil the late evolution of the universe, because some of the Galileon's configurations have negative energy densities while on some other the NEC is broken so that the energy density in them will still grow with time even after the end of reheating. In [39], only the particular Genesis solution was studied, while bouncing trajectories in the model were first investigated in [47]. The full cosmological phase space was investigated in [41], demonstrating that, without a reheating mechanism, all trajectories eventually evolve to the configurations where the sound speed becomes zero, so that the original effective field theory (EFT) becomes infinitely strongly coupled. This strong-coupling regime occurs before the would-be Big Rip future singularity. This conclusion also applies to the Genesis solution which turns out to be a separatrix in the cosmological phase space of the Galilean Genesis.

Perturbations propagating around the Genesis solution initially do so with a sound speed equal to that of light, slowing down as the expansion becomes significant, and eventually vanishing. In these higher-derivative scalar theories, superluminal propagation is common, see e.g. [48]. While its presence does not necessarily signify the existence of causal paradoxes or problems on the level of EFT see e.g. [49-53] ${ }^{7}$, it does mean that any EFT where a connected part of the phase space features superluminality cannot have an ultra-violet (UV) completion which is local, Lorentz invariant and corresponds to some weakly coupled heavier particles [48]. Thus such theories cannot have a standard Wilsonian UV completion, but may still be completed in a non-standard way, via e.g. classicalization introduced in [61,62] for such derivatively coupled scalars. Moreover, it was argued [63-65] that classicalization may only work provided that superluminality is possible.

To avoid the superluminality of perturbations and give a hope for a Wilsonian UV completion, in [66], Subluminal Galilean Genesis was introduced. An additional parameter in the action ensures that the sound speed on the Genesis trajectory can be arbitrarily small, while the substantial features of the original Galilean Genesis are preserved. This should imply that in other parts of the phase space, at least those close to the Genesis trajectory, the sound speed would be similarly reduced, in principle removing any such potential superluminality.

In this paper, we extend our phase-space analysis presented in [41] to the Subluminal Galilean Genesis model.

First, in section 3 we analytically prove that any configuration of a spatially-flat Friedmann universe filled solely by the Galileon with the action and parameters from Subluminal Galilean Genesis [66] has a subluminal sound speed. In fact, this is true even of the original Galilean Genesis model [39].

However, as a result of the imperfect nature of the fluid [67] described by the scalar field or kinetic mixing / braiding of the scalar with the metric, the local properties of the Galileon do not just depend on the local scalar-field variables, but also on the local Ricci tensor and, through the Einstein equations, on all other external matter species present locally. In particular, this implies that the sound speed of the Galileon changes in the presence of other matter species. Using this unusual property, we show analytically in section 4 that for all admissible parameter values of the Subluminal Galilean Genesis model, there are always cosmological configurations which feature superluminal propagation given the addition of an appropriate external positive energy density with a normal equation of state $0<w<1$. Moreover, perturbations around these configurations are not ghosts and some of these configurations correspond to the same values of the local scalar-field variables available in the phase space of the Subluminal Galilean Genesis without any external matter. In particular, these configurations can be obtained by a continuous transformation from the Genesis trajectory. We illustrate our analytic proofs with numerical computations.

Thus as such, none of the Subluminal Galilean Genesis models is free of superluminality in the presence of external matter and by the arguments of [48] cannot enjoy a standard Wilsonian UV completion.

\footnotetext{
${ }^{7}$ For other recent studies of superluminality in various derivatively coupled theories see e.g. [54-60].
} 


\section{$2 \quad$ Model and Main Equations}

We study the class of models for the Galileon scalar-field $\pi$, minimally coupled to gravity $g_{\mu \nu}$, the dynamics of which are given by the action introduced in $[39,66]^{8}$

$$
S_{\pi}=\int \mathrm{d}^{4} x \sqrt{-g}\left[-f^{2} e^{2 \pi}(\partial \pi)^{2}+\gamma \frac{\beta}{2}(\partial \pi)^{4}+\gamma(\partial \pi)^{2} \square \pi\right],
$$

where $\square=g^{\mu \nu} \nabla_{\mu} \nabla_{\nu}$, with $\nabla_{\mu}$ representing the covariant derivative while

$$
\beta=1+\alpha,
$$

is a dimensionless parameter bound to be in the range $1 \leq \beta<4$, and another dimensionless parameter

$$
\gamma=\left(\frac{f}{\Lambda}\right)^{3} \gg 1
$$

is a combination of constants $f$ and $\Lambda$ of dimension one. The positive parameter $\alpha$ was introduced in [66] and represents the only difference between the system (2.1) and the so-called Conformal Galileon used to drive the Galilean Genesis scenario in [39], where $\alpha=0$, and which was also studied in context of a bouncing cosmology in e.g. [41, 47, 68]. The reason for the introduction of the positive $\alpha$ in [66] is the "robust subluminality" around the Genesis solution. We will mostly use $\beta$ to simplify formulae.

It is useful to perform the following field redefinition

$$
\pi=\ln \left(\frac{\phi}{f}\right)
$$

so that one obtains a form of the action where the scalar field $\phi$ has standard dimensions,

$$
S_{\phi}=\int \mathrm{d}^{4} x \sqrt{-g}\left[-(\partial \phi)^{2}+\gamma\left(\frac{\beta-2}{2}\right) \frac{(\partial \phi)^{4}}{\phi^{4}}+\gamma \frac{(\partial \phi)^{2}}{\phi^{2}} \frac{\square \phi}{\phi}\right],
$$

and the action has just two dimensionless parameters $\beta$ and $\gamma$. In particular, from this form of the action, it is clear that no single physical observable related purely to the evolution of this system can depend on $\Lambda$ and $f$ separately but only on their ratio given by $\gamma$. However, if there are non-minimal but non-universal direct couplings to some external fields / matter through

$$
g_{\mu \nu}^{\text {matter }}=e^{2 \pi} g_{\mu \nu}=\left(\frac{\phi}{f}\right)^{2} g_{\mu \nu},
$$

as was proposed in [39] for a spectator field $\sigma$, then $f$ plays a role of the coupling constant to this type of external matter, see [29]. This coupling is not only needed to generate fluctuations as in [39] but also to reheat and possibly exit from the super-inflationary regime [46]. Note that the coupling cannot be universal for all matter, since the model (2.1) would then just correspond to inflation written in a conformally rescaled metric. Therefore there should be at least some matter species which do not couple to the Galileon directly through (2.5) but only through gravity. Further we note that the system simplifies for $\beta=2$.

The dimensionless constant $\gamma$ can be moved in front of the whole action if one rescales the coordinates as

$$
x^{\mu} \rightarrow \gamma^{1 / 2} y^{\mu}, \text { so that } S_{\phi} \rightarrow \gamma S_{\phi}
$$

Note that the Einstein-Hilbert action scales in the same way $S_{\mathrm{EH}} \rightarrow \gamma S_{\mathrm{EH}}$. Hence the dynamics of the Galileon field $\phi$ interacting with gravity is completely independent of $\gamma$ in the absence of other

\footnotetext{
${ }^{8}$ Contrary to $[39,66]$ we use the signature convention $(+,-,-,-)$, so that the sign in front of the first term is "wrong" - it corresponds to a ghost.
} 
fields. In a realistic universe there should be other matter species, e.g. radiation or cold dark matter. If we assume that those are not coupled to the Galileon directly but only through gravity one should rescale their energy density (and pressure) by

$$
\rho \rightarrow \rho / \gamma
$$

so that, after this rescaling, $\gamma$ disappears from all equations. Therefore the rescaled energy density is allowed to be larger than unity but should be parametrically smaller than $\gamma$ in order to avoid physical transplanckian energy densities.

\subsection{Some properties of models with Kinetic Gravity Braiding}

Both the versions of the action for Galilean Genesis, (2.1) and (2.4), belong to the same class ${ }^{9}$

$$
S_{\varphi}=\int \mathrm{d}^{4} x \sqrt{-g}[K(\varphi, X)+G(\varphi, X) \square \varphi],
$$

where $K$ and $G$ are arbitrary functions of $\varphi$ and where

$$
X=\frac{1}{2} g^{\mu \nu} \partial_{\mu} \varphi \partial_{\nu} \varphi
$$

We are going to work with both actions (2.1) and (2.4), hence, to make the paper self-contained, we will list the main dynamical equations and stability criteria valid for a general theory of type (2.8). After that one can use these equations by substituting $\varphi \rightarrow \pi$ or $\phi$ and the corresponding $K$ and $G$. All these equations can be found with derivations in this form in [33, 67]. In a cosmological setup, for these systems, it is convenient to use hydrodynamical notation and analogy introduced in [67].

The diffusivity measuring the kinetic mixing / braiding of $\varphi$ with the metric is

$$
\kappa \equiv 2 X G_{, X}
$$

where ()$_{, X} \equiv \partial() / \partial X$. The effective mass per shift-charge / chemical potential is

$$
m \equiv \partial_{t} \varphi \equiv \dot{\varphi} .
$$

The pressure of this scalar imperfect fluid is

$$
\mathcal{P}=K-m^{2} G_{, \varphi}-\kappa \dot{m},
$$

while the density of shift charges (which are not conserved for the systems studied in this paper) is

$$
n=K_{, m}-2 m G_{, \varphi}+3 H \kappa,
$$

where $H$ is the Hubble parameter. The corresponding energy density is

$$
\mathcal{E}=m n-\mathcal{P}-\kappa \dot{m}=\varepsilon+3 H m \kappa,
$$

where

$$
\varepsilon=m\left(K_{, m}-m G_{, \varphi}\right)-K,
$$

is a "perfect part" of the energy density which is independent of the expansion or Hubble parameter. In the spatially flat Friedmann universe we have ${ }^{10}$

$$
H^{2}=\frac{1}{3}(\mathcal{E}+\rho)=\kappa m H+\frac{1}{3}(\varepsilon+\rho),
$$

\footnotetext{
${ }^{9}$ This class of models was introduced in [33] as kinetic gravity braiding and then slightly later in [40].

${ }^{10}$ Throughout the paper we use the reduced Planck units where $M_{\mathrm{Pl}}=\left(8 \pi G_{\mathrm{N}}\right)^{-1 / 2}=1$.
} 
where $\rho$ is the energy density in external matter species. We have assumed that these species are not coupled to the Galileon directly, so that the corresponding equation of motion is the continuity equation $\dot{\rho}+3 H(\rho+p)=0$, where $p$ is the their pressure. The second Friedmann equation is

$$
\dot{H}=-\frac{1}{2}(\mathcal{E}+\rho+\mathcal{P}+p)=\frac{1}{2}(\kappa \dot{m}-n m-(\rho+p)) .
$$

The equation of motion for the scalar $\varphi$ is

$$
\dot{m} D+3 n\left(H-\frac{1}{2} \kappa m\right)+\mathcal{E}_{, \varphi}=\frac{3}{2} \kappa(\rho+p),
$$

where

$$
D=\frac{\varepsilon_{, m}}{m}+3 H \kappa_{, m}+\frac{3}{2} \kappa^{2} .
$$

The partial derivatives are all taken at constant Hubble parameter $H$. The perturbations are not ghosts provided $D>0$. Finally the sound speed is given by the formula

$$
c_{\mathrm{s}}^{2}=\frac{n+\dot{\kappa}+\kappa(H-\kappa m / 2)}{D m} .
$$

Fluids with Kinetic Gravity Braiding (including the Galileon fluid) are conservative - they do not have a notion of entropy - but they are nonetheless imperfect fluids. One of the peculiarities of these systems is that the local properties not only depend on the fluid variables, which in the cosmological context are $\varphi$ and $m$, but also on the expansion (Hubble parameter) and on the external matter energy density $\rho$ and pressure $p$, see equation of motion (2.18). In particular, the condition for the absence of ghosts (2.19) explicitly depends on $H$, i.e. on the external energy density, while the sound speed not only depends on the Hubble parameter, i.e. on $\rho$, but also directly on the external pressure $p$, because of the $\dot{\kappa}$ term in (2.20) and the structure of the equation of motion (2.18). Thus, contrary to $D$, the sound speed also depends on the external equation of state $w=p / \rho$. This dependence of local properties of fluids with Kinetic Gravity Braiding on external $\rho$ and $p$ reveals a similarity of these fluids with open systems.

These properties are crucial for our analysis and the appearance of the superluminality.

\section{Robust subluminality without external matter}

In this section, we will work with the $\pi$ field and action (2.1) without any external matter. First of all, we eliminate $\gamma$ by rescaling (2.6) so that the time variable which enters both $\dot{\pi}$ and $H$

$$
t=\gamma^{1 / 2} \tau,
$$

and consequently the rescaled variables $m$ and $h$ are defined by

$$
m=\dot{\pi} \gamma^{1 / 2} \text {, and } h=H \gamma^{1 / 2} .
$$

We will denote differentiation with respect to this new time variable with a prime, ()$^{\prime} \equiv \mathrm{d} / \mathrm{d} \tau$. Note that we use the same notation, $m$, as in (2.11) for the non-rescaled effective mass of a unit shift-charge. The cosmological dynamics are described by the system of three first order differential equations (2.11), (2.17) and (2.18) with the constraint (2.16), where the external energy density and pressure are taken to be zero in all equations. Thus the phase space is a $2 \mathrm{~d}$ hypersurface in the space $(\pi, m, h)$. For a similar analysis of a phase-space geometry, see e.g. [69]. The phase space cannot be uniquely projected into the plane $(\pi, m)$, which corresponds to a natural parametrisation of Galileon states, see Fig. 1. Instead, following our paper [41], it is useful to describe dynamics in phase space $(m, h)$ by solving the Friedmann equation (2.16) with respect to $e^{2 \pi}$ :

$$
e^{2 \pi}=\frac{3 m^{4} \beta+12 h m^{3}-6 h^{2}}{2 m^{2}} .
$$




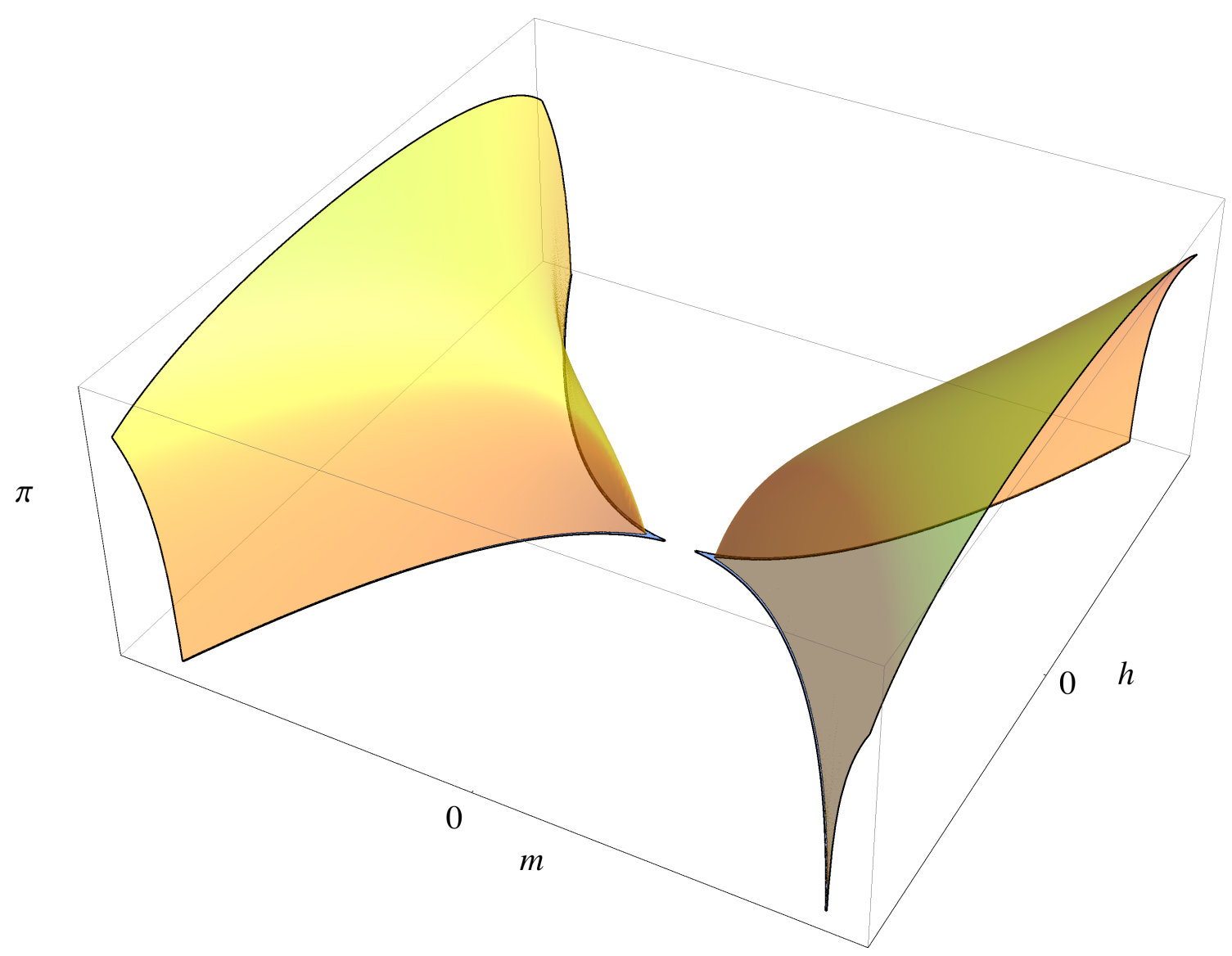

Figure 1. The yellow hypersurface represents the phase space for the cosmological dynamics of the Subluminal Galilean Genesis given by the system of first order differential equations (2.11), (2.17) and (2.18) with the constraint (2.16). The external energy density and pressure are taken to be zero in all equations. The numerical resolution is insufficient to plot the origin of the phase space.

Thus the expression on the r.h.s. must be strictly positive. Wherever the above function is negative, it corresponds to a region of the phase space which is dynamically inaccessible. The advantage of the variables $(m, h)$ is that one avoids in this way branches in the square root and related folding of the phase space present for $(\pi, m)$ description, see discussion in the subsection 4.2.

In the variables $(m, h)$ the evolution of the system is given by equations (2.18) and (2.17) which after elimination of $\pi$ and rescaling can be written as

$$
\begin{aligned}
& m^{\prime}=m \frac{\left[12 h^{2}-m^{2}\left(m^{2}-1\right) \beta+h m\left(4-6 m^{2}+\beta\right)\right] m^{3}-2 h^{2}(3 h+m)}{2 h^{2}+m^{4}\left(2 m^{2}+\beta\right)}, \\
& h^{\prime}=\frac{\left[12 h^{2} m^{2}+\left(8 h m^{3}-4 h^{2}+m^{4} \beta\right)(1+\beta)+m^{4} \beta\right] m^{4}-12 h^{4}}{2\left(2 h^{2}+m^{4}\left(2 m^{2}+\beta\right)\right)} .
\end{aligned}
$$

We have plotted this phase space for a number of values of $\beta$ in Fig. 2.

This system evolves in the region $\Phi(m, h)$ restricted because of (3.3) to:

$$
m^{4} \beta+4 h m^{3}-2 h^{2}>0 .
$$

Thus the phase space $\Phi(m, h)$ is located between two curves

$$
h_{ \pm}(m)=m^{2}\left[m \pm \sqrt{m^{2}+\frac{\beta}{2}}\right] .
$$


Further, the absence of ghosts requires the positiveness of $D$ from (2.19) and provides the bound

$$
\frac{m^{2}}{3} D=2 h^{2}+m^{4}\left(2 m^{2}+\beta\right)>0,
$$

which is always satisfied for $\beta=1+\alpha>1$. On Fig. 2, we have marked the inaccessible regions in deep red with the boundaries defined by (3.6). There are no ghosty regions.

The general formula (2.20) for the sound speed translates for $c_{\mathrm{s}}^{2}(m, h)$ to

$$
c_{\mathrm{s}}^{2}=1-\frac{12}{(D m)^{2}} m \mathscr{P}(m, h),
$$

where the polynomial cubic in $h$

$$
\mathscr{P}(m, h)=8 h^{3}+2 h^{2} m\left(1-4 m^{2}+\beta\right)+4 h m^{4}\left(2 m^{2}-1\right)+m^{5}\left(4 m^{4}+5 m^{2} \beta+(\beta-1) \beta\right),
$$

defines whether the sound speed is less or larger than the speed of light. Subluminality corresponds to $m \mathscr{P}(m, h)>0$. For each $m$, the polynomial $\mathscr{P}(m, h)$ can have maximum 3 real roots $h_{\mathrm{s}}(m)$. The number of roots, when solved for $h$, is defined by the sign of the discriminant $\mathscr{D}(\alpha, m)$,

$$
\begin{aligned}
& -\frac{\mathscr{D}(\alpha)}{32 m^{8}}=2144 m^{10}+16 m^{8}[125+203 \alpha]+2 m^{6}[339+\alpha(1066+915 \alpha)]+ \\
& +4 m^{4}[38+\alpha(126+\alpha(194+115 \alpha))]+m^{2}[32+\alpha(116+\alpha(154+\alpha(119+47 \alpha)))]+ \\
& +\alpha(1+\alpha)(2+\alpha)^{3} .
\end{aligned}
$$

This is the only formula for which the parameter $\alpha=\beta-1$ is more convenient. The polynomial on the r.h.s. has only even powers of $m$ and is manifestly positive for all non-negative $\alpha$. Thus the discriminant $\mathscr{D}(\alpha, m)$ of the polynomial $\mathscr{P}(m, h)$ is always negative for all $m \neq 0$ and non-negative $\alpha$, whereas $\mathscr{D}(\alpha, 0)=0$. Therefore, for $m \neq 0$, the polynomial $\mathscr{P}(m, h)$ has only one real root $h_{\mathrm{s}}(m)$ which one can find analytically, but the expression for which is by far too long to be suitable for any analysis $^{11}$. Now we can evaluate the value of $m \mathscr{P}\left(m, h_{ \pm}(m)\right)$ at the borders $h_{ \pm}(m)$ of the phase space $\Phi(m, h)$ given by $(3.6)$. For $h_{-}(m)$ we obtain

$$
m \mathscr{P}\left(m, h_{-}(m)\right)=\frac{8 m^{6}}{\left(2 m^{2}+\beta\right)^{2}}\left(\xi^{4}-4 \xi^{3}+9 \xi^{2}-8 \xi+4\right),
$$

where $\xi=m / \sqrt{m^{2}+\beta / 2}$. This function $m \mathscr{P}\left(m, h_{-}(m)\right)$ is always non-negative since the polynomial in $\xi$ is positive for $\xi=0$ and has four complex roots $\frac{1}{2}(2-i \pm \sqrt{-5-4 i})$ and the complex conjugates. For the other boundary, $h_{+}(m)$, we have

$$
m \mathscr{P}\left(m, h_{+}(m)\right)=\frac{8 m^{6}}{\left(2 m^{2}+\beta\right)^{2}}\left(\xi^{4}+4 \xi^{3}+9 \xi^{2}+8 \xi+4\right),
$$

which is also always non-negative, because the polynomial in $\xi$ has four complex roots of the form $\frac{1}{2}(-2-i \pm \sqrt{-5-4 i})$ and the complex conjugates.

This means that the single real root $h_{\mathrm{s}}(m)$ of $\mathscr{P}(m, h)$ is not located in the phase space and $m \mathscr{P}(m, h) \geq 0$ not only on the boundaries $h_{ \pm}(m)$ but the whole cosmological phase space $\Phi(m, h)$. Therefore, for all $\alpha \geq 0$, we conclude that the system is subluminal on all cosmological configurations. In this sense we have proven a strong version of the desired property 6 on page 2 from [66].

We have illustrated this in Fig. 2. The roots $h_{\mathrm{s}}(m)$ of $(3.9)$ are the boundaries of the blue regions. As can be seen for $\beta \geq 1(\alpha \geq 0)$ there is only one such boundary and it always occurs inside the deep red dynamically inaccessible region, as we have proven. Only in the case of $\beta=0(\alpha=-1)$, which is not part of the parameter space of Subluminal Galilean Genesis, a new region of superluminality appears: now, for a range of values of $m$, there are three real roots $h_{\mathrm{s}}(m)$ delineating the boundaries of these superluminal regions. Two of these roots lie inside the phase space.

\footnotetext{
${ }^{11}$ Note that for $\alpha<0$ this situation changes so that for sufficiently small $m$ the polynomial $\mathscr{P}(m, h)$ has 3 real roots and there is always a superluminal region, see the lower right panel in Fig. 2.
} 


\section{Superluminality with external matter}

In this section we consider cosmology with the Galileon in the presence of external matter with a standard equation of state $w=p / \rho=$ const, e.g. dust or radiation. We will restrict our attention to matter satisfying the Strong Energy Condition (SEC) and the Dominant Energy Condition (DEC) so that $-1 / 3 \leq w \leq 1$. The main purpose of this section is to prove that when such external mater is added into the game there are some regions in cosmological phase space where the fluctuations of the Galileon propagate faster than light. Moreover, in some of these regions the perturbations are not ghosty so that these configurations should belong to the same effective filed theory (EFT) which describes the regions where the perturbations are subluminal and those where the external matter is negligible or absent at all.

\section{1 $(m, h, \rho)$ coordinates and a proof of general superluminality}

First, similarly to the previous section, we can proceed by working in the phase space $(m, h, \rho)$ where $\rho$ is rescaled as in (2.7). One can eliminate $\pi$ by solving the first Friedmann equation (2.16) with respect to $\pi$ so that

$$
e^{2 \pi}=\frac{2 \rho+3 m^{4} \beta+12 h m^{3}-6 h^{2}}{2 m^{2}} .
$$

Therefore the phase space is restricted by

$$
\frac{2}{3} \rho-2 h^{2}+4 h m^{3}+m^{4} \beta>0 .
$$

The perturbations of the Galileon are not ghosts when $D$ from (2.19) is positive which provides the inequality

$$
m^{2} D=6 h^{2}+6 m^{6}+3 m^{4} \beta-2 \rho>0 .
$$

We would like to stress that, contrary to k-essence, the coefficient $D$ explicitly depends on the external energy density $\rho$. Thus a sufficiently large positive amount of the external energy density can turn the perturbations of the Galileon into ghosts. Moreover, in the presence of $\rho$ there are always ghosts close enough to the origin of the phase space, $m=h=0$.

From the above inequalities, it follows that the external energy density should be located

$$
3 h^{2}+\frac{3}{2} m^{4} \beta-6 h m^{3}<\rho<3 h^{2}+\frac{3}{2} m^{4} \beta+3 m^{6} .
$$

This is only possible for such $(m, h)$ that

$$
m\left(m^{3}+2 h\right)>0 .
$$

In particular this works for $m>0$ and $h>0$. For the sound speed (2.20) one obtains

$$
c_{\mathrm{s}}^{2}=1-\frac{4}{(D m)^{2}} m\left[3 \mathscr{P}(m, h)-\rho\left(8 h+2 m(\beta+1)+m^{3}(1+3 w)\right)\right],
$$

where $\mathscr{P}(m, h)$ is given by (3.9) and is the same polynomial as in the previous section. For $m>0$ and $8 h+2 m(\beta+1)+m^{3}(1+3 w)>0$, the sound speed is larger than the speed of light provided

$$
\rho>\rho_{\text {sup }}=\frac{3 \mathscr{P}(m, h)}{8 h+2 m(1+\beta)+m^{3}(1+3 w)} .
$$

If, contrary to the matter-free case, dynamics (4.1) allow for such $(m, h)$ that $m \mathscr{P}(m, h)<0$, then a positive amount of external matter which satisfies the no-ghost condition (4.2) will make the perturbations superluminal. 
Now let us prove that it is always possible to find such $\rho$. It is convenient to introduce a "probe" energy density

$$
\rho_{\lambda}(m, h)=3 h^{2}+\frac{3}{2} m^{4} \beta+\lambda m^{6},
$$

We restrict the numerical parameter $\lambda$ to $0<\lambda<3$. This restriction ensures that probe energy density is always positive and fits into the borders (4.3) for $m>0$ and $h>0$. On this probe energy density we have

$$
c_{\mathrm{s}}^{2}=1+\frac{Z(m, h)}{2 m^{6}(\lambda-3)^{2}},
$$

where

$$
\begin{aligned}
& Z(m, h)=18 h^{2}(3+w)+8 h m\left[3(1+\beta)+2 m^{2}(\lambda-3)\right]+ \\
& +m^{2}\left[12 \beta+m^{2}(9(w-3) \beta+4(1+\beta) \lambda)+2 m^{4}(\lambda(1+3 w)-12)\right] .
\end{aligned}
$$

$\lambda=3$ is the boundary of the ghosty region on which the sound speed (4.5) diverges. For normal matter with $w>-3$, the polynomial $Z$ as a function of $h$ is a convex parabola. Thus for sufficiently large $h$ this function $Z$ is always positive. On the other hand, for given $(m, h)$, we can choose $\lambda$ arbitrarily close to 3, i.e. arbitrarily close to the ghosty region. Thus we conclude that the sound speed of the Galileon can not only be superluminal but can, indeed, acquire arbitrarily large positive values. We would like to stress that by construction the perturbations are not ghosts on these configurations. This conclusion only uses the positivity of $\beta$ which is needed for the positivity of $\rho_{\lambda}$.

One may think that the superluminality can only correspond to large $h$ but in fact there are always regions around $h=0$ where the sound speed is larger than 1 . Indeed, in that case

$$
\frac{3}{2} m^{4} \beta<\rho<\rho_{\mathrm{gh}}=\frac{3}{2} m^{4} \beta+3 m^{6},
$$

and

$$
c_{\mathrm{s}}^{2}=1-\frac{4 m^{4}\left[3 m^{4}\left(4 m^{4}+5 m^{2} \beta+(\beta-1) \beta\right)-\rho\left(2(\beta+1)+m^{2}(1+3 w)\right)\right]}{\left(6 m^{6}+3 m^{4} \beta-2 \rho\right)^{2}} .
$$

Thus, for $w>-1 / 3$ which are considering here, one has to require that

$$
\rho>\rho_{\text {sup }}=\frac{3 m^{4}\left(4 m^{4}+5 m^{2} \beta+(\beta-1) \beta\right)}{2(\beta+1)+m^{2}(1+3 w)},
$$

and there are $\rho$ realising this regime provided $\rho_{\text {sup }}<\rho_{\text {gh }}$. This happens for those $m$ for which

$$
6 m^{4}(w-1)+m^{2}(4-\beta(5-3 w))+4 \beta>0 .
$$

The latter inequality is manifestly satisfied for sufficiently small $m^{2}$ because $\beta \geq 1$. Thus the superluminality without ghosts can also happen for small $m$ and $h$ or in other words for $\rho \ll 1$.

\section{2 $(\phi, m, \rho)$ coordinates and induced superluminality of original configurations}

The disadvantage of choosing the phase space in terms of $(m, h, \rho)$ in section 4.1 is that adding external energy density $\rho$ while keeping both $m$ and $h$ fixed forces the value of the scalar field $\pi$ to change. Thus, in some sense, the new configuration including $\rho$ does not correspond to the initial one. To avoid this, in this section, we will consider the phase space in terms of the coordinates $(\phi, m, \rho)$, where $\phi$ is given by (2.3), $m=\pi^{\prime}=\phi^{\prime} / \phi$, i.e. is still given by (3.2) and $\rho$ is again rescaled as in (2.7). However, we will see this is much more complicated as a result of the fact that this three-dimensional phase space is double folded, because of the square root

$$
\Omega_{\rho}=\sqrt{6\left[2 \rho+m^{2}\left(3 \beta m^{2}+6 m^{4}-2 \phi^{2}\right)\right]},
$$


in the solution of the Friedmann equation (2.16)

$$
h=m^{3}+\frac{1}{6} \Omega_{\rho} .
$$

Here $\Omega_{\rho}=\Omega(\phi, m, \rho)$ is defined in such a way that the sign of $\Omega_{\rho}$ changes when the argument of the square root evolves trough zero. Only regions where the argument of the square root in $\Omega_{\rho}$ is positive correspond to physically available configurations, which result in a condition on $\rho$,

$$
\rho \geq \phi^{2}-\frac{3}{2} m^{2}\left(\beta+2 m^{2}\right) .
$$

In this discussion, we are only considering normal matter and parameter ranges relevant to the subluminal Galilean Genesis model, i.e. we will assume in what follows that

$$
\begin{aligned}
-1 / 3 & \leq w \leq 1, \quad w=\text { const } \\
1 & \leq \beta \leq 4 .
\end{aligned}
$$

Let us introduce the configurations of the Galileon which are dynamically allowed without any external matter:

$$
\Gamma=(\phi, m): \text { such configurations that } \phi^{2} \leq \frac{3}{2} m^{2}\left(\beta+2 m^{2}\right) .
$$

These configurations are exactly those which we have studied in the section 3 , just in different variables. In the rest of this section we will only consider these configurations $\Gamma$. If we add any positive $\rho \geq 0$ to any of these configurations $\Gamma$, it is still dynamically allowed, since (4.16) holds. The addition of the positive energy density $\rho$ opens up the phase space - allows one to probe new cosmological configurations $(\phi, m)$ violating the condition (4.18).

For the positivity of $\rho$ we have to require that

$$
\rho \text { positive: } \quad \Omega_{\rho}^{2}>\Omega_{0}^{2}=6 m^{2}\left[3 \beta m^{2}+6 m^{4}-2 \phi^{2}\right] .
$$

On configurations $\Gamma$ we always have $\Omega_{0}^{2}>0$.

As we have shown before, in the section 3 all configurations $\Gamma$ have subluminal sound speed when there is no external matter. In the subsection 4.1 we have showed that superluminality takes place somewhere on available and not ghosty phase space. However, these regions could not be available without external matter - so that for $(\phi, m)$ the condition (4.18) is violated. Our main goal now is to prove that around some of the configurations $\Gamma$ the sound speed can become superluminal on the addition of external matter, but the perturbations remain not ghosty.

The perturbations are not ghosts when $D$ from (2.19) is positive, which provides in our current variables the inequality

$$
\frac{\phi^{2} D}{2}=m\left(\Omega_{\rho}-\Omega_{\text {ghosts }}\right)>0, \text { where } \Omega_{\text {ghosts }} \equiv \frac{\phi^{2}-3 m^{2}\left(\beta+3 m^{2}\right)}{m} .
$$

Therefore the condition for the absence of ghosts reduces to

$$
\begin{array}{ll}
\text { No ghosts: } & \Omega_{\rho}>\Omega_{\text {ghosts }}, \text { for } m>0, \\
& \Omega_{\rho}<\Omega_{\text {ghosts }}, \text { for } m<0,
\end{array}
$$

Note that $\Omega_{\text {ghosts }}(\Gamma)>0$ for $m<0$ and $\Omega_{\text {ghosts }}(\Gamma)<0$ for $m>0$. Further it is convenient to express

$$
\Omega_{\text {ghosts }}=-\frac{\Omega_{0}^{2}+18 m^{4}\left(\beta+4 m^{2}\right)}{12 m^{3}} .
$$

Hence, on $\Gamma$ and for sufficiently small $m^{2}$ one always obtains $\Omega_{\text {ghosts }}^{2}(\Gamma)>\Omega_{0}^{2}(\Gamma)$. 
The sound speed $(2.20)$ as a function on phase space $(\phi, m, \rho)$ is given by

$$
c_{\mathrm{s}}^{2}(\phi, m, \rho)=1-\frac{m \mathscr{R}_{w}\left(\phi, m, \Omega_{\rho}\right)}{3 \phi^{4} D^{2}},
$$

where

$$
\begin{aligned}
& \mathscr{R}_{w}\left(\phi, m, \Omega_{\rho}\right)=(7-3 w) \Omega_{\rho}^{2} m+16 \Omega_{\rho}\left(3 m^{2}\left(\beta+3 m^{2}\right)-\phi^{2}\right)+ \\
& +6 m\left[3 m^{2}\left(4 \beta^{2}+3(9+w) \beta m^{2}+6(7+w) m^{4}\right)-2 \phi^{2}\left(2(1+\beta)+3(3+w) m^{2}\right)\right] .
\end{aligned}
$$

The superluminality is present in regions where $m \mathscr{R}_{w}\left(\phi, m, \Omega_{\rho}\right)<0$. $\mathscr{R}_{w}\left(\phi, m, \Omega_{\rho}\right)$ depends on $\rho$ only through $\Omega_{\rho}$, with respect to which $m \mathscr{R}_{w}\left(\phi, m, \Omega_{\rho}\right)$ is a convex parabola. Thus superluminality is only possible if the roots $\Omega_{w \pm}$ of $\mathscr{R}_{w}\left(\phi, m, \Omega_{\rho}\right)$ are real. Given that, in order to have superluminality, $\Omega_{\rho}$ must lie between these real roots:

Superluminality exists: $\quad \Omega_{w-}<\Omega_{\rho}<\Omega_{w+}$

$$
\Omega_{w \pm}=\frac{8}{7-3 w}\left[\Omega_{\text {ghosts }} \pm \sqrt{\Omega_{\text {ghosts }}^{2}+\Sigma}\right], \Omega_{w \pm} \in \mathbb{R},
$$

where $\Omega_{\text {ghosts }}$ is defined in $(4.20)$ and

$$
\Sigma=-\frac{(7-3 w)}{16}\left[9 m^{2}\left[(\beta-1) \beta+(7 \beta-2) m^{2}+12 m^{4}\right]+\left[2(1+\beta)+3(3+w) m^{2}\right] \frac{\Omega_{0}^{2}}{4 m^{2}}\right]
$$

with $\Omega_{0}$ given by (4.19). Now we will prove that there are such $(\phi, m)$ from $\Gamma$ that these roots exist and that at least some $\Omega_{\rho}$ between these roots do not have ghosts and have $\rho>0$.

For $\Omega_{w \pm}$ of (4.25) to be real, we need to require that

$$
\Omega_{\text {ghosts }}^{2}+\Sigma \geq 0
$$

For our chosen parameters $w$ and $\beta,(4.17), \Sigma(\Gamma)<0$, on all configurations $\Gamma$. Therefore, if $\Omega_{w \pm}$ exist somewhere on $\Gamma$, then for $m>0$ we have $\Omega_{w-}(\Gamma) \leq \Omega_{w+}(\Gamma)<0$ whereas for $m<0$ these roots are located $0<\Omega_{w-}(\Gamma) \leq \Omega_{w+}(\Gamma)$.

To avoid ghosts as in (4.21), we have to require that

$$
\begin{array}{ll}
\Omega_{w+}=\frac{8}{7-3 w}\left[\Omega_{\text {ghosts }}+\sqrt{\Omega_{\text {ghosts }}^{2}+\Sigma}\right]>\Omega_{\text {ghosts }}, & \text { for } m>0, \\
\Omega_{w-}=\frac{8}{7-3 w}\left[\Omega_{\text {ghosts }}-\sqrt{\Omega_{\text {ghosts }}^{2}+\Sigma}\right]<\Omega_{\text {ghosts }}, & \text { for } m<0 .
\end{array}
$$

For configurations $\Gamma$ and normal matter these are equivalent to the condition

$$
\text { Superluminal \& no ghosts: } \Sigma>\Omega_{\text {ghosts }}^{2}\left(\left(\frac{1+3 w}{8}\right)^{2}-1\right)
$$

which is a stronger condition than the condition (4.27) for the existence of roots $\Omega_{w \pm}$ and which implies

$$
\Omega_{w-}<\Omega_{\text {ghosts }}<\Omega_{w+} .
$$

Therefore if (4.29) holds and $m^{2}$ is sufficiently small, one can always adjust $\Omega_{\rho}$ to be sufficiently close to $\Omega_{\text {ghosts }}$ so that $\Omega_{0}^{2}<\Omega_{\rho}^{2}<\Omega_{\text {ghosts }}^{2}$ and therefore have superluminality with $\rho>0$ and no ghosts.

Now let us find simple sufficient conditions on $(\phi, m)$ from $\Gamma$ to satisfy $(4.29)$, proving that there are accessible superluminal configurations. 
The inequality (4.29) is equivalent to

$$
\mathcal{F}\left(\phi^{2}\right)=(3+w) \phi^{4}+B \phi^{2}+C>0,
$$

where

$$
B=2 m^{2}\left[4-(5+3 w) \beta-3 m^{2}(3+w)\right],
$$

and

$$
C=3 m^{4}\left[3 m^{4}(5 w-1)+12 m^{2} w \beta+(1+3 w) \beta^{2}\right] .
$$

If for some range of $m$ there are no real roots for the equation $\mathcal{F}\left(\phi^{2}\right)=0$, then the inequality (4.31) (or equivalently (4.29)) holds for all $\phi$ and in particular for such $\phi$ that the matter-free configuration belongs to $\Gamma$, (4.18). Inequality (4.31) holds for all $\phi$, provided the quadratic equation for $\phi^{2}$ does not have positive solutions, in particular, if the discriminant is negative, i.e. if

$$
S\left(m^{2}\right)=18 m^{4}(1-w)(3+w)-3 m^{2}(3+w)(4+(3 w-5) \beta)-4(5+3 w-2 \beta) \beta+8<0 .
$$

Let us consider sufficiently small $m^{2}$. For any $\beta \geq 1$ and

$$
w>w_{\beta}=\frac{2\left(\beta+\beta^{-1}\right)-5}{3},
$$

we obtain $S(0)<0$, so that for sufficiently small $m^{2}$ we have $S\left(m^{2}\right)<0$. In particular for $\beta=1$ we have $w_{1}=-1 / 3$ thus any SEC satisfying matter creates a small region close to $m=0$ such that the sound speed is superluminal and yet the perturbations are not ghosts. For $\beta=2$ this translates into $w_{2}=0$ so that reasonable matter would always create a superluminal region. Whereas, for $\beta=4$, which is the maximal interesting value of this parameter from [66] we have $w_{4}=7 / 6$ which would violate the DEC and which is not an equation of state available for standard matter. The limiting equation of state $w_{\beta}$ corresponds to radiation when

$$
\beta_{\mathrm{rad}}=\frac{3+\sqrt{5}}{2} \simeq 2.62
$$

and to ultra-stiff equation of state $w_{\beta}=1$ when

$$
\beta_{\text {stiff }}=2+\sqrt{3} \simeq 3.73
$$

Thus for $1 \leq \beta \leq \beta_{\text {stiff }}$ the addition of standard matter creates a superluminal but not ghosty region at least for those configurations from $\Gamma$ which have sufficiently small $m$.

Now we can look for more general larger $m$. Since $S\left(m^{2}\right)$ of $(4.31)$ is a convex parabola, if we require that $S\left(m^{2}\right)=0$ has at least one positive root $m^{2}$, then there will be a range of $m$ where the inequality (4.34) is satisfied and where consequently (4.29) holds for all $\phi$. These roots are given by

$$
m_{ \pm}^{2}=\frac{12-15 \beta+w(4+(4+3 w) \beta) \pm \sqrt{(3+w) Y(w, \beta)}}{12(1-w)(3+w)},
$$

where

$$
Y(w, \beta)=(1+w)(11+3 w(3 w-4)) \beta^{2}-8(1+w)(9 w-5) \beta+80 w-16,
$$

which is a quadratic polynomial in $\beta$ and cubic in $w$. We now must ensure that both $Y>0$ and $m_{+}^{2}>0$. For normal matter $(1+w)(11+3 w(3 w-4))>0$, thus for $Y$ to be positive, the parameter $\beta$ must be larger than

$$
\beta_{*}(w)=\frac{4\left[w(4+9 w)+2 \sqrt{3(3+w)\left(1-w^{2}\right)(1-3 w)}-5\right]}{(1+w)(11+3 w(3 w-4))},
$$


for normal matter with $w<1 / 3$, and $\beta$ can be arbitrary for $w>1 / 3$. It is easy to check that $\beta_{*}<1$ for all $w$ we consider, thus $Y$ is always positive for our set of parameters (4.17).

For $0 \leq w \leq 1 / 3$ one obtains that $m_{-}^{2}$ is always negative whereas $m_{+}^{2}$ is positive for either

$$
1 \leq \beta \leq 2 \text { and } 0 \leq w \leq 1 / 3
$$

or for

$$
2 \leq \beta<\beta_{\mathrm{rad}} \text { and } \frac{(\beta-2)(2 \beta-1)}{3 \beta}<w \leq \frac{1}{3}
$$

If we allow for matter with an ultra-relativistic equation of state $1 / 3 \leq w \leq 1$ then

$$
\beta_{\text {rad }} \leq \beta<\beta_{\text {stiff }} \text { and } \frac{(\beta-2)(2 \beta-1)}{3 \beta}<w<1
$$

Thus we have proved that for any model with $\beta<\beta_{\text {stiff }} \simeq 3.73$ one can add a positive amount of external energy energy density with $0<w<1$ to make the sound speed of the perturbations around at least some original configurations larger than the speed of light. We presume that this statement also holds for $\beta_{\text {stiff }}<\beta<4$. But to prove this it would require a more cumbersome analysis e.g. requiring the roots $\phi^{2}$ of $\mathcal{F}\left(\phi^{2}\right)=0$ to be negative. We should also stress that we have only constructed one type of configurations with superluminality. We have not performed an exhaustive search to classify all such configurations.

\section{$5 \quad$ Results Summary and Discussion}

In the case when there is no external matter, we have analytically proved in section 3 that for a spatially flat Friedmann universe the perturbations of the Galileon are never superluminal. To illustrate our results we have plotted phase flows in coordinates $(m, h)$ (which are rescaled $(\dot{\pi}, H))$ in Fig. 2. The red regions are dynamically inaccessible as the r.h.s. of (3.3) is negative, but there are no ghosty regions in the phase space at all. The trajectories correspond to solutions of (3.4). The Galilean Genesis trajectory is marked as the thick salmon line originating at the origin. The regions of superluminality are coloured in deep blue. We can clearly see that for $\beta \geq 1$ they never occur in the phase space. Indeed there is no superluminality anywhere in the phase space even in the original Galilean Genesis scenario of [39].

The situation changes upon adding external matter with positive energy density $\rho$, coupled to the scalar $\pi$ only through gravity. In section 4 we have an analytic proof that there are regions in the phase space where the perturbations are propagating faster than light. In Fig. 3, we have plotted a two-dimensional slice through the now three-dimensional phase space $(m, h, \rho)$, keeping $\rho$ fixed. Adding external energy density opens up a ghosty region surrounding the origin of the $(m, h)$ axes, which we have plotted in yellow. The boundary of this region is a pressure singularity at which the sound speed diverges. The neighbourhood of this boundary can emit trajectories and can be approached by trajectories. Therefore there is always a superluminal region, containing arbitrarily high sound speeds, surrounding this ghosty region. This is true for arbitrarily small positive external energy densities $\rho$ and for all values of $\beta$ relevant for the Subluminal Galilean Genesis scenario. Adding external energy density allows for new cosmological configurations of the scalar field $(\pi, \dot{\pi})$, opening up the phase space in this sense. The addition of positive energy density with a normal equation of state can sometimes transform a cosmological configuration of the scalar field, into a configuration without ghosts but with superluminal propagation of perturbations.

Further, we would like to mention that, similarly to the Ghosts Condensate [70] or the simplest $k$ inflation [71], the systems described by (2.1) or (2.4) do not possess any physically acceptable Lorentzinvariant vacuum. This breakdown is acceptable for a theory effectively describing a hydrodynamics of an (im)perfect fluid. However, as a field theory it is far beyond the Standard Model - the QFT which describes the current state of knowledge in particle physics. The appearance of ghosts around $X=0$ 

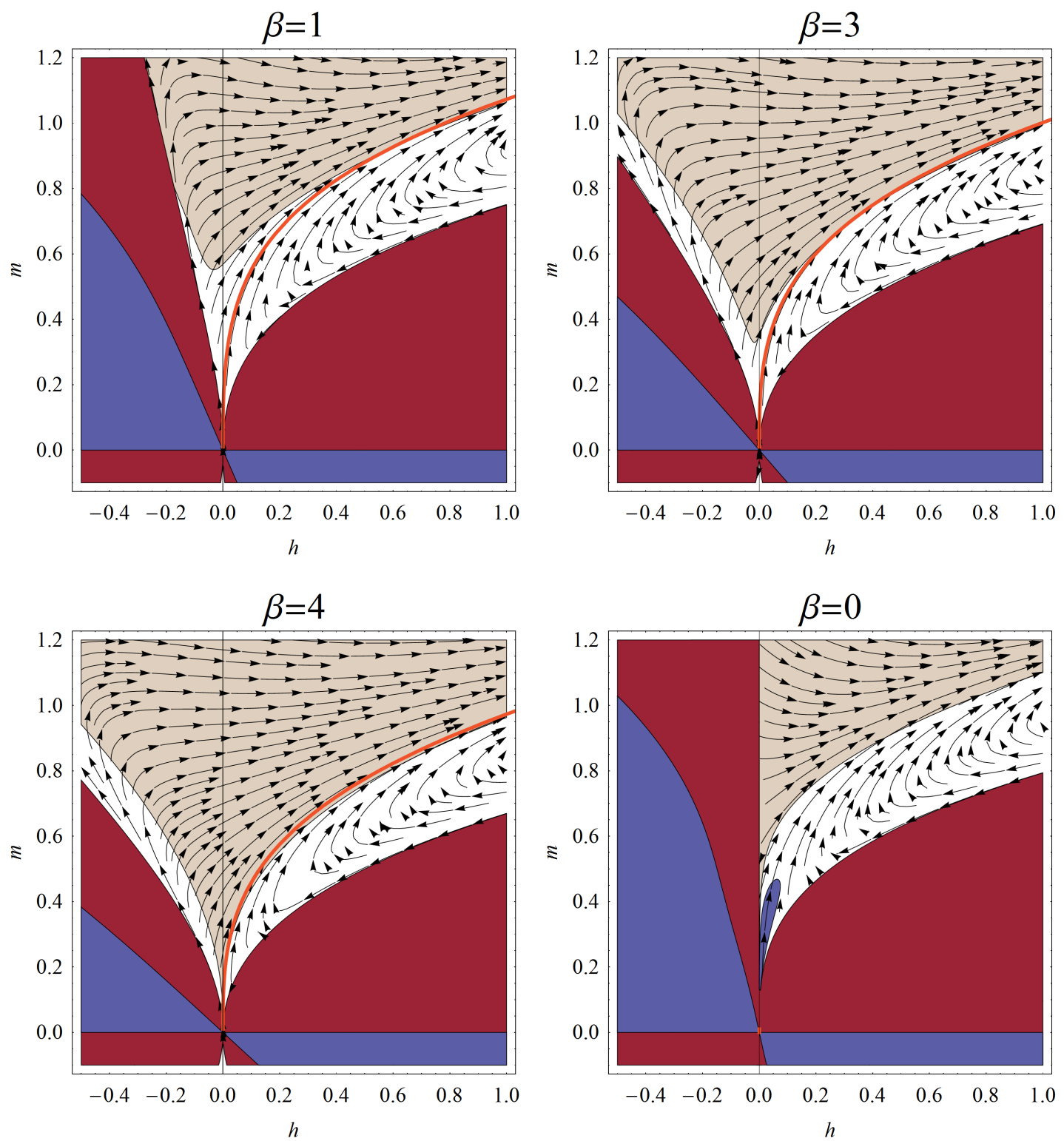

Figure 2. Phase portrait for the Subluminal Galilean Genesis system (3.4) for various values of the parameter $\beta \equiv 1+\alpha$ with no external matter present. The axes are rescaled coordinates $h=(f / \Lambda)^{3 / 2} H$, $m=(f / \Lambda)^{3 / 2} \dot{\pi}$. The deep red region is dynamically inaccessible, the blue corresponds to configurations where the sound speed is superluminal, the sandy region- to those configurations where $c_{\mathrm{s}}^{2}<0$ and gradient instabilities are present. Healthy trajectories evolve through the white regions and have been illustrated with flow lines. The Galilean Genesis trajectory discussed in $[39,66]$ is a separatrix and has been explicitly singled out and marked as the thick salmon-coloured line. Nowhere in this phase space is there a region where the perturbations are ghosty.

For $\beta \geq 1$, the accessible phase space never has any superluminality anywhere. This includes the original Galilean Genesis model with $\beta=1$. As $\beta$ increases, the sound speeds are reduced and eventually for $\beta=4$ the sandy region with imaginary sound speed reaches to the origin of the phase space which should correspond to a singular Minkowski space. For $\beta<1$, an accessible superluminal region appears at the origin and grows in extent with decreasing $\beta$. 


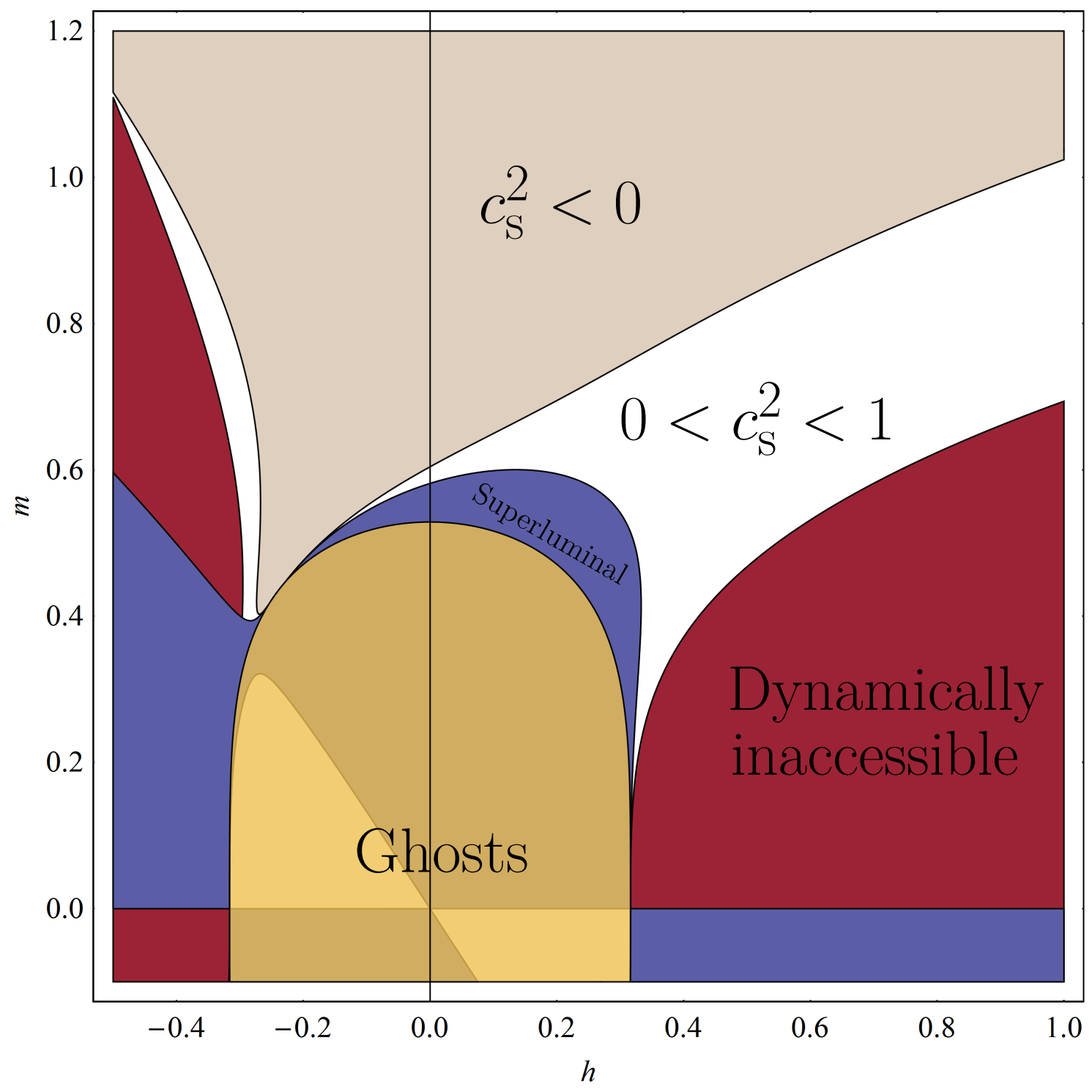

Figure 3. A slice through the phase space of the Subluminal Galilean Genesis model with $\beta=2$ in the presence of external radiation $(w=1 / 3)$ with rescaled energy density $\rho=0.3$. The axes are rescaled coordinates $h=(f / \Lambda)^{3 / 2} H, m=(f / \Lambda)^{3 / 2} \dot{\pi}$. The whole phase space is $(m, h, \rho)$. The deep red regions are dynamically inaccessible; inside the yellow region the perturbations of $\pi$ are ghosts. The sandy region has $c_{\mathrm{s}}^{2}<0$ while in the blue the sound speed is superluminal. The sound speed diverges as the boundary of the yellow ghosty region.

The blue superluminal region to the top right of the yellow ghosty region is accessible to trajectories and has arbitrarily high sound speed close to the ghosty region. It appears for arbitrarily small values of $\rho$ and even at $h=0$. We demonstrate this analytically in section 4 . 
separates the phase space into two disconnected sets which correspond to two different EFTs - one for configurations with spacelike derivatives and another one for the timelike case. Note that a smooth dynamical evolution from one set to another, i.e. from timelike to spacelike derivatives or vice versa, is physically impossible, because of the pressure singularity preventing these transitions [33, 41, 67] in these theories without a healthy Lorentz-invariant vacuum. This is obviously the case for the standard representation of fluid dynamics through fields, see e.g. [72]. Close to normal vacuum, where the energy density of the fluid and particle density are zero, the fluid description should break down in any case and one is forced to use kinetic theory. In field-theoretical language this breakdown would correspond to some pathology of the EFT - to an infinitely large strong-coupling scale or ghosts or unacceptable gradient instability. This property is crucial for the EFTs which allow for superluminal propagation. Indeed, if such EFT can only exist for timelike gradients of some scalar field, then one can always declare this field to be the time coordinate so that the resulting spacetime is stably causal and any causal paradoxes are impossible by definition, see e.g. [51].

On the other hand, exactly the absence of a physically acceptable Lorentz-invariant vacuum in the context of an EFT implies that one cannot use this EFT to describe a transition from inflation (or from some Genesis stage of the early universe as in [39, 66]) to standard hot Big Bang cosmology.

Properties of a possible UV completion of an EFT should depend on all states / configurations this EFT is able to describe. It is not enough to look at a given state or given trajectory and its small neighbourhood. Indeed, why should the EFT be inapplicable further away if nothing prevents it from operating there? Only in the case when the phase space (or Hilbert space) has different unconnected regions can one claim that there are different EFT's describing these regions separately. These regions can be separated by states or configurations with ghosts or gradient instabilities or some classical singularities or infinitely strong coupling. For example, this is the case for perfect fluids where the same Lagrangian could be formally used for spacelike gradients. In some of these configurations with spacelike gradients, superluminal propagation may be possible. This, however, would not at all imply that one cannot find weakly coupled particles building the fluid corresponding to the timelike gradients for which there is no superluminal propagation.

In this paper we have only studied external matter which is not coupled to the Galileon. Indeed, this matter should unavoidably be present in the model. However, we do not think that a non-minimal direct coupling (2.5) would change the situation.

Another potential way to avoid our conclusion is to prove that the energy density $\rho_{\mathrm{s}}(\phi, m)$ needed to induce superluminality around a configuration $(\phi, m)$ brings the EFT away from its region of validity. However, this is hard to check, since the strong coupling scale $\mu$ (for wave vectors) generically depends on all cosmological phase space coordinates, so that $\mu=\mu(\phi, m, \rho)$. On dimensional grounds, one would expect that the limiting external energy density for a given $(\phi, m)$ should be provided by the solution of the equation $\rho=c_{\mathrm{s}}(\phi, m, \rho) \mu^{4}(\phi, m, \rho)$, where $c_{\mathrm{S}}$ is the sound speed. Unfortunately there is no self-consistent derivation of $\mu$ as a function of an arbitrary cosmological configuration $(\phi, m, \rho)$. This analysis is definitely interesting and important but involves the calculation of the cubic action for the cosmological perturbations in this general non-slow-roll setup. This task goes beyond the scope of our paper. Moreover, it follows from our analysis that the corresponding $\rho_{\mathrm{s}}(\phi, m)$ is not necessary parametrically large. If the addition of such an external $\rho$ can invalidate the EFT it could imply that reheating might also lie outside of the region of validity of this EFT.

It would also be interesting to investigate whether one could modify the theory in such a way that superluminal propagation would be impossible on all configurations with timelike gradients in the presence of any matter with a normal equation of state. In such a case, the superluminality could only occur for some unrealistic external matter. However, given the properties above, this could be impossible and would definitely be a cumbersome and ambitious task. But only such strong property could give a hope for a standard Wilsonian UV completion without a need for something less standard like classicalization [61, 62].

We expect that the situation with UV completion is typical for all recently rediscovered scalar field theories able to violate the NEC. 


\section{Acknowledgments}

The work of DAE is supported in part by the DOE under DE-SC0008016 and by the Cosmology Initiative at Arizona State University. IS is supported by the DFG through TRR33 "The Dark Universe". IS would like to thank the CERN Theory Group for hospitality during the preparation of this paper. The work of AV is supported by ERC grant BSMOXFORD no. 228169. AV is thankful to the organisers and staff of the Cook's Branch Spring Workshop, under the auspices of the Mitchell Institute for Fundamental Physics and Astronomy at Texas A\&M University for a warm hospitality during the final stages of this project.

\section{References}

[1] V. F. Mukhanov and G. V. Chibisov, "Quantum Fluctuation and Nonsingular Universe. (In Russian)," JETP Lett. 33 (1981) 532-535.

[2] G. Chibisov and V. F. Mukhanov, "Galaxy formation and phonons," Mon. Not. Roy. Astron. Soc. 200 (1982) 535-550. .

[3] S. Hawking, "The Development of Irregularities in a Single Bubble Inflationary Universe," Phys. Lett. B115 (1982) 295.

[4] A. A. Starobinsky, "Dynamics of Phase Transition in the New Inflationary Universe Scenario and Generation of Perturbations," Phys.Lett. B117 (1982) 175-178.

[5] A. H. Guth and S. Pi, "Fluctuations in the New Inflationary Universe," Phys. Rev. Lett. 49 (1982) 1110-1113.

[6] J. M. Bardeen, P. J. Steinhardt, and M. S. Turner, "Spontaneous Creation of Almost Scale - Free Density Perturbations in an Inflationary Universe," Phys.Rev. D28 (1983) 679.

[7] V. F. Mukhanov, "Gravitational Instability of the Universe Filled with a Scalar Field," JETP Lett. 41 (1985) 493-496.

[8] M. Sasaki, "Large Scale Quantum Fluctuations in the Inflationary Universe," Prog.Theor.Phys. 76 (1986) 1036.

[9] V. F. Mukhanov, "CMB, quantum fluctuations and the predictive power of inflation," arXiv: astro-ph/0303077.

[10] V. Mukhanov, "Quantum Cosmological Perturbations: Predictions and Observations," arXiv:1303.3925 [astro-ph.CO].

[11] A. A. Starobinsky, "Relict Gravitation Radiation Spectrum and Initial State of the Universe. (In Russian)," JETP Lett. 30 (1979) 682-685.

[12] A. H. Guth, "The Inflationary Universe: A Possible Solution to the Horizon and Flatness Problems," Phys.Rev. D23 (1981) 347-356.

[13] A. D. Linde, "A New Inflationary Universe Scenario: A Possible Solution of the Horizon, Flatness, Homogeneity, Isotropy and Primordial Monopole Problems," Phys.Lett. B108 (1982) 389-393.

[14] A. Albrecht and P. J. Steinhardt, "Cosmology for Grand Unified Theories with Radiatively Induced Symmetry Breaking," Phys.Rev.Lett. 48 (1982) 1220-1223.

[15] A. A. Starobinsky, "A New Type of Isotropic Cosmological Models Without Singularity," Phys.Lett. B91 (1980) 99-102.

[16] K. Sato, "First Order Phase Transition of a Vacuum and Expansion of the Universe," Mon.Not.Roy.Astron.Soc. 195 (1981) 467-479.

[17] D. Kazanas, "Dynamics of the Universe and Spontaneous Symmetry Breaking," Astrophys.J. 241 (1980) L59-L63.

[18] R. Brout, F. Englert, and E. Gunzig, "The Causal Universe," Gen.Rel.Grav. 10 (1979) 1-6.

[19] É. B. Gliner, "The Vacuum-Like State of a Medium and Friedman Cosmology," Soviet Physics Doklady 15 (Dec., 1970) 559. 
[20] Planck Collaboration Collaboration, P. Ade et al., "Planck 2013 results. XVI. Cosmological parameters," arXiv:1303.5076 [astro-ph.CO].

[21] A. Borde, A. H. Guth, and A. Vilenkin, "Inflationary space-times are incompletein past directions," Phys.Rev.Lett. 90 (2003) 151301, arXiv:gr-qc/0110012 [gr-qc].

[22] S. Dubovsky, T. Gregoire, A. Nicolis, and R. Rattazzi, "Null energy condition and superluminal propagation," JHEP 0603 (2006) 025, arXiv:hep-th/0512260 [hep-th].

[23] P. Creminelli, M. A. Luty, A. Nicolis, and L. Senatore, "Starting the Universe: Stable Violation of the Null Energy Condition and Non-standard Cosmologies," JHEP 0612 (2006) 080, arXiv:hep-th/0606090 [hep-th].

[24] P. Creminelli and L. Senatore, "A smooth bouncing cosmology with scale invariant spectrum," JCAP 0711 (2007) 010, arXiv:hep-th/0702165.

[25] C. Cheung, P. Creminelli, A. L. Fitzpatrick, J. Kaplan, and L. Senatore, "The Effective Field Theory of Inflation," JHEP 0803 (2008) 014, arXiv:0709.0293 [hep-th].

[26] P. Creminelli, G. D’Amico, J. Norena, and F. Vernizzi, "The Effective Theory of Quintessence: the $w<-1$ Side Unveiled," JCAP 0902 (2009) 018, arXiv:0811.0827 [astro-ph].

[27] M. A. Luty, M. Porrati, and R. Rattazzi, "Strong interactions and stability in the DGP model," JHEP 0309 (2003) 029, arXiv:hep-th/0303116 [hep-th] .

[28] A. Nicolis and R. Rattazzi, "Classical and quantum consistency of the DGP model," JHEP 0406 (2004) 059, arXiv:hep-th/0404159 [hep-th].

[29] A. Nicolis, R. Rattazzi, and E. Trincherini, "The galileon as a local modification of gravity," Phys. Rev. D79 (2009) 064036, arXiv:0811.2197 [hep-th].

[30] C. Deffayet, G. Esposito-Farese, and A. Vikman, "Covariant Galileon," Phys. Rev. D79 (2009) 084003, arXiv:0901.1314 [hep-th].

[31] C. Deffayet, S. Deser, and G. Esposito-Farese, "Generalized Galileons: All scalar models whose curved background extensions maintain second-order field equations and stress-tensors," Phys.Rev. D80 (2009) 064015, arXiv:0906.1967 [gr-qc].

[32] C. de Rham and A. J. Tolley, "DBI and the Galileon reunited," JCAP 1005 (2010) 015, arXiv:1003.5917 [hep-th].

[33] C. Deffayet, O. Pujolas, I. Sawicki, and A. Vikman, "Imperfect Dark Energy from Kinetic Gravity Braiding," JCAP 1010 (2010) 026, arXiv:1008.0048 [hep-th].

[34] C. Deffayet, X. Gao, D. Steer, and G. Zahariade, "From k-essence to generalised Galileons," Phys.Rev. D84 (2011) 064039, arXiv:1103.3260 [hep-th].

[35] T. Kobayashi, M. Yamaguchi, and J. Yokoyama, "Generalized G-inflation: Inflation with the most general second-order field equations," Prog.Theor.Phys. 126 (2011) 511-529, arXiv:1105. 5723 [hep-th].

[36] G. W. Horndeski, "Second-order scalar-tensor field equations in a four-dimensional space," Int. J. Theor. Phys. 10 (1974) 363-384.

[37] I. Sawicki and A. Vikman, "Hidden Negative Energies in Strongly Accelerated Universes," Phys.Rev. D87 (2013) 067301, arXiv:1209.2961 [astro-ph.CO].

[38] A. Nicolis, R. Rattazzi, and E. Trincherini, "Energy's and amplitudes' positivity," JHEP 05 (2010) 095, arXiv:0912.4258 [hep-th].

[39] P. Creminelli, A. Nicolis, and E. Trincherini, "Galilean Genesis: An Alternative to inflation," JCAP 1011 (2010) 021, arXiv:1007.0027 [hep-th].

[40] T. Kobayashi, M. Yamaguchi, and J. Yokoyama, "G-inflation: Inflation driven by the Galileon field," Phys.Rev.Lett. 105 (2010) 231302, arXiv:1008.0603 [hep-th].

[41] D. A. Easson, I. Sawicki, and A. Vikman, "G-Bounce," JCAP 1111 (2011) 021, arXiv:1109.1047 [hep-th]. 
[42] K. Hinterbichler, A. Joyce, J. Khoury, and G. E. Miller, "DBI Realizations of the Pseudo-Conformal Universe and Galilean Genesis Scenarios," JCAP 1212 (2012) 030, arXiv:1209.5742 [hep-th].

[43] K. Hinterbichler, A. Joyce, J. Khoury, and G. E. Miller, "DBI Genesis: An Improved Violation of the Null Energy Condition," arXiv:1212.3607 [hep-th].

[44] A. A. Starobinsky, "Future and origin of our universe: Modern view," Grav. Cosmol. 6 (2000) 157-163, arXiv:astro-ph/9912054 [astro-ph].

[45] R. R. Caldwell, M. Kamionkowski, and N. N. Weinberg, "Phantom Energy and Cosmic Doomsday," Phys. Rev. Lett. 91 (2003) 071301, arXiv:astro-ph/0302506.

[46] L. Levasseur Perreault, R. Brandenberger, and A.-C. Davis, "Defrosting in an Emergent Galileon Cosmology," Phys. Rev. D84 (2011) 103512, arXiv:1105.5649 [astro-ph.C0].

[47] T. Qiu, J. Evslin, Y.-F. Cai, M. Li, and X. Zhang, "Bouncing Galileon Cosmologies," JCAP 1110 (2011) 036, arXiv:1108.0593 [hep-th].

[48] A. Adams, N. Arkani-Hamed, S. Dubovsky, A. Nicolis, and R. Rattazzi, "Causality, analyticity and an IR obstruction to UV completion," JHEP 0610 (2006) 014, arXiv:hep-th/0602178 [hep-th].

[49] J.-P. Bruneton, "On causality and superluminal behavior in classical field theories. Applications to k-essence theories and MOND-like theories of gravity," Phys. Rev. D75 (2007) 085013, arXiv: gr-qc/0607055.

[50] J. U. Kang, V. Vanchurin, and S. Winitzki, "Attractor scenarios and superluminal signals in k-essence cosmology," Phys. Rev. D76 (2007) 083511, arXiv:0706.3994 [gr-qc].

[51] E. Babichev, V. Mukhanov, and A. Vikman, "k-Essence, superluminal propagation, causality and emergent geometry," JHEP 02 (2008) 101, arXiv:0708.0561 [hep-th].

[52] R. Geroch, "Faster Than Light?," arXiv:1005.1614 [gr-qc].

[53] C. Burrage, C. de Rham, L. Heisenberg, and A. J. Tolley, "Chronology Protection in Galileon Models and Massive Gravity," JCAP 1207 (2012) 004, arXiv:1111.5549 [hep-th].

[54] J. Evslin and T. Qiu, "Closed Timelike Curves in the Galileon Model," JHEP 1111 (2011) 032, arXiv:1106.0570 [hep-th].

[55] J. Evslin, "Stability of Closed Timelike Curves in a Galileon Model," JHEP 1203 (2012) 009, arXiv:1112.1349 [hep-th].

[56] K. Hinterbichler, A. Nicolis, and M. Porrati, "Superluminality in DGP," JHEP 0909 (2009) 089, arXiv:0905.2359 [hep-th].

[57] A. Gruzinov, "All Fierz-Paulian massive gravity theories have ghosts or superluminal modes," arXiv:1106.3972 [hep-th].

[58] C. de Rham, G. Gabadadze, and A. J. Tolley, "Comments on (super)luminality," arXiv:1107.0710 [hep-th].

[59] S. Deser and A. Waldron, "Acausality of Massive Gravity," Phys. Rev. Lett. 110 (2013) 111101, arXiv:1212.5835 [hep-th].

[60] P. de Fromont, C. de Rham, L. Heisenberg, and A. Matas, "Superluminality in the Bi- and MultiGalileon," arXiv:1303.0274 [hep-th].

[61] G. Dvali, G. F. Giudice, C. Gomez, and A. Kehagias, "UV-Completion by Classicalization," JHEP 1108 (2011) 108, arXiv:1010.1415 [hep-ph].

[62] G. Dvali and D. Pirtskhalava, "Dynamics of Unitarization by Classicalization," Phys.Lett. B699 (2011) 78-86, arXiv:1011.0114 [hep-ph].

[63] G. Dvali, "Classicalize or not to Classicalize?," arXiv:1101.2661 [hep-th].

[64] G. Dvali, A. Franca, and C. Gomez, "Road Signs for UV-Completion," arXiv:1204.6388 [hep-th].

[65] A. Vikman, "Suppressing Quantum Fluctuations in Classicalization," Europhys. Lett. 101 (2013) 34001, arXiv: 1208.3647 [hep-th]. 
[66] P. Creminelli, K. Hinterbichler, J. Khoury, A. Nicolis, and E. Trincherini, "Subluminal Galilean Genesis," JHEP 1302 (2013) 006, arXiv:1209.3768 [hep-th].

[67] O. Pujolas, I. Sawicki, and A. Vikman, "The Imperfect Fluid behind Kinetic Gravity Braiding," JHEP 1111 (2011) 156, arXiv:1103.5360 [hep-th].

[68] M. Osipov and V. Rubakov, "Galileon bounce after ekpyrotic contraction," arXiv:1303.1221 [hep-th].

[69] G. N. Felder, A. V. Frolov, L. Kofman, and A. D. Linde, "Cosmology with negative potentials," Phys.Rev. D66 (2002) 023507, arXiv:hep-th/0202017 [hep-th].

[70] N. Arkani-Hamed, H.-C. Cheng, M. A. Luty, and S. Mukohyama, "Ghost condensation and a consistent infrared modification of gravity," JHEP 05 (2004) 074, arXiv: hep-th/0312099.

[71] C. Armendariz-Picon, T. Damour, and V. F. Mukhanov, "k-Inflation," Phys. Lett. B458 (1999) 209-218, arXiv:hep-th/9904075.

[72] B. F. Schutz, "Perfect Fluids in General Relativity: Velocity Potentials and a Variational Principle," Phys.Rev. D2 (1970) 2762. 